\title{
Magnetic Field-Trapping Properties of Melt-Processed Y123 Bulk Superconductors Activated by Pulsed-Field Magnetizing Method
}

\author{
Tetsuo Oka*, Yukimasa Yamada, Daichi Ishiduka, Jun Ogawa, Satoshi Fukui, Takao Sato, \\ Kazuya Yokoyama* and Marco Langer** \\ Niigata University, 8050 Ikarashi-Ninocho, Nishi-Ward, Niigata, 950-2181 Japan \\ Fax: 81-25-262-7666, e-mail: okat@eng.niigata-u.ac.jp \\ * Ashikaga Institute of Technology, 268-1 Ohmae-cho, Ashikaga, Tochigi, 326-8558 Japan \\ Fax: 81-284-62-0609, e-mail: k-yokoyama@ashitech.ac.jp \\ ** IFW Dresden, Helmholzstr., 20-01069 Dresden, Germany \\ e-mail: m.langer@ifw-dresden.de
}

The magnetic flux invasion into the $\mathrm{Y}-\mathrm{Ba}-\mathrm{Cu}-\mathrm{O}$-based high temperature superconducting bulk magnets were precisely evaluated during the pulsed field magnetization processes. Various bulk magnets with different contents of $\mathrm{Y}_{2} \mathrm{BaCuO}_{5}$ (Y211) particles were fabricated so as to examine how the Y211 concentrations affect the flux invasion behaviors. The flux motions and the resultant trapped field densities were monitored at the surface of each bulk sample at $30.6 \mathrm{~K}$. As a result, the applied fields at which the magnetic flux began to invade the sample increased with increasing Y211 contents. This means that the pinning centers are promoted with increasing nominal Y211 addition. According to the evolutional profiles during the pulsed-field applications, the flux-penetration ratio gradually promoted with increasing applied fields, whereas the flux-trapping ratio decreased. This implies that the heat generation due to the flux motion seriously affects the field-trapping ability. The anomalous behaviors for the sample, which has displaced position of seed crystal, showed us more effective flux-trapping than usual, exhibiting the higher flux-penetration and flux-trapping ratios at lower applied-field than those of other samples. This suggests a possible high-field activation method with preferential flux invasion due to the different microstructures in the bulk magnet.

Key words: bulk superconductor, melt-process, magnetic pulsed-field, flux-penetration, trapped field, heat generation

\section{INTRODUCTION}

Largely-grown high temperature superconducting (hereafter abbreviated as HTS) materials have a characteristic feature so as to act as we call the trapped-field magnets or bulk magnets, generating intense magnetic fields of more than several $\mathrm{T}$ on the sample surfaces [1, 2]. The highest field-trapping performance reached over $17 \mathrm{~T}$ by the process what we call as the field cooling (FC) method conducted at $29 \mathrm{~K}$, in which the bulk magnet was activated in the static magnetic field by superconducting solenoid-type magnet [3]. On the other hand, some investigations including our previous studies have been performed on the pulsed-field magnetization (PFM) methods to develop more compact and easier processes than FC method [4, 5]. According to their reports, the fact that the field-trapping performance through the PFM process is usually inferior to those by the FC activation is attributed to the local heating in the material, which substantially is caused by the rapid motion of the applied magnetic flux even in its superconducting state $[6,7]$.

The keys for high field-trapping are the temperature controlling [8] and the consecutive magnetic pulsed-field application in the magnetizing processes [9]. The highest performance of $5.2 \mathrm{~T}$ was reported so far by Fujishiro et al. in MMPSC activation method with the precise analysis on the trapped-field distributions throughout the process $[10,11]$. Our past studies clarified that the previous trapped-field distributions in a bulk magnet affect the magnetic flux behaviors in the following pulsed field applications [12]. Therefore, it is definitely important to clarify the behaviors of the invading flux into the bulk magnets during the PFM processes to make them trap intense magnetic fields and to enhance their field-trapping ability.

It is well-known that the melt-processed bulk magnets show the heterogeneous microstructures according to the presence of non-superconducting $\mathrm{Y}_{2} \mathrm{BaCuO}_{5}$ (Y211) particles which must contribute to the enhancement of $J_{\mathrm{c}}$ [13]. The invasion behaviors of the quantumized fluxoids in the superconducting materials are strongly affected by the $J_{\text {c }}$ values which strongly relate to the presence the Y211 particles. In this paper, the bulk magnets with various contents of Y211 particles were fabricated so as to examine how the concentrations of Y211 particles affect the field-penetration behaviors when intense magnetic pulsed fields are applied to the samples. And, we attempt to examine the various flux-trapping properties and the flux motions through the discussions on the data of trapped-flux densities and temperature changes.

2. EXPERIMENTAL PROCEDURE

2.1 Sample preparation and structural analysis 


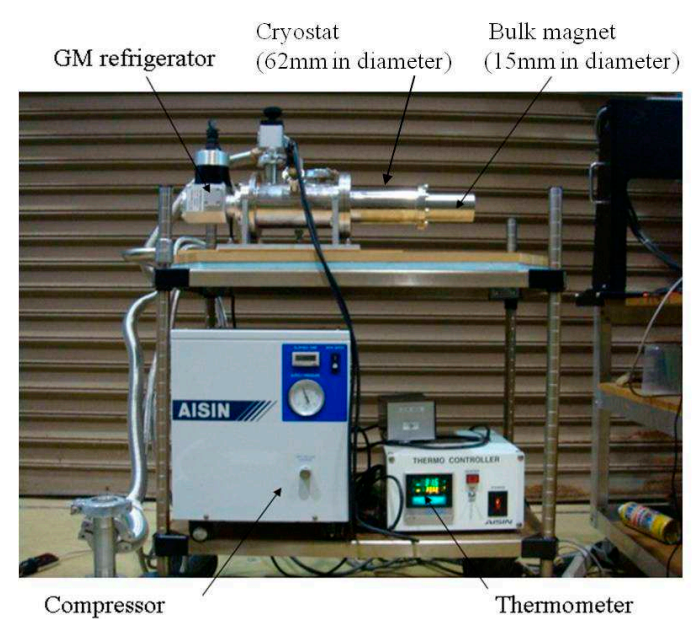

Fig. 1. Strong magnetic field generator equipped with GM refrigerator which cools the bulk magnet to $30.6 \mathrm{~K}$ in the vacuum chamber.

The raw materials of $\mathrm{YBa}_{2} \mathrm{Cu}_{3} \mathrm{O}_{7-\delta}(\mathrm{Y} 123)$ and $\mathrm{Y} 211$ powders (supplied by Toshima Seisakusho Co., the mean size of the particles is $1 \mu \mathrm{m}$ ) were mixed in the proper ratios with $1 \mathrm{wt} \% \mathrm{CeO}_{2}$ addition in the zirconia mortar and pressed into the pellets with dimensions of $20 \mathrm{~mm}$ in diameter and $10 \mathrm{~mm}$ in thickness. The Y211 nominal contents were chosen as $20,25.8,40,50 \%$ in molar ratios. The precursors were heat-treated in the cold seeding method with the $\mathrm{Nd}-\mathrm{Ba}-\mathrm{Cu}-\mathrm{O}$ thin films on the $\mathrm{MgO}$ crystal substrates (THEVA Co.) as seed crystals. In the melt-process, the precursors were heated to $1050{ }^{\circ} \mathrm{C}$, and then gradually solidified from the peritectic temperature with the cooling rate $0.25^{\circ} \mathrm{C} / \mathrm{h}$ to let the crystals grow large. The post annealing was operated in the temperature range of $500-300{ }^{\circ} \mathrm{C}$ for 167 hours in the flowing oxygen atmosphere. The structural analysis was operated with use of electron probe micro analyzer (EPMA, JEOL Ltd, JXA-8621MX). We investigated the compositional varieties of $\mathrm{Y} 211$ on the EPMA mapping on $\mathrm{Y}\left(\mathrm{L}_{\alpha}\right)$.

\subsection{Pulsed-field magnetization procedure}

Figure 1 shows the experimental setup of the PFM technique. The system is mainly composed of a cryostat with the size of $68 \mathrm{~mm}$ in diameter, a GM refrigerator
(AISIN SEIKI CO., GR-103) which cools the bulk magnet to $30.6 \mathrm{~K}$ in the vacuum chamber, a compressor to supply the pressurized helium gas to the cooler, and a temperature controller. An illustrated structure of the total system is shown in Fig. 2(a) with the electrical circuit which is equipped with a condenser bank of 120 $\mathrm{mF}$ (Nihon Denji Sokki Co., SBV-10124), a shunt resister, and an oscilloscope. Intense magnetic fields were generated by feeding currents from the condenser bank to the pulse coil which activates the bulk magnet. The pulse coil is dipped in the liquid nitrogen vessel to reduce the resistance by cooling it to $77 \mathrm{~K}$ [12]. The coil constant is $0.925 \mathrm{mT} / \mathrm{A}$. The iron yokes are coupled with the bulk magnet to attract and lead the magnetic flux into the sample during the field application. As Fig. 2(b) shows, a Hall sensor (F. W. Bell, BHT 921) was attached just at the centre of the sample surface so as to measure the flux motion during the PFM operation. A fine $\mathrm{Cu}$-Constantan thermocouple, the size of which is $0.07 \mathrm{~mm}$ in diameter, was glued on it at the position in the growth sector region. Figure 2(b) shows the positions of the Hall sensor and the thermocouple. In the PFM process, we applied various magnitudes of the successive pulsed-fields ranging from 2.78 to $6.10 \mathrm{~T}$. We measured the temperature changes, the evolutional magnetic flux density profiles during the magnetizing procedures, and the resultant trapped flux densities after the serial activation processes.

\section{RESULTS AND DISCUSSION}

3.1 HTS bulk magnets and their structural analysis

Figure 3 shows the topside-views of the bulk magnet samples, which were fabricated by the melt-process with various amounts of Y211 additions. The sample size is approximately $15 \mathrm{~mm}$ in diameter. One sees the apparent growth sector boundaries, which indicate their characteristic "X-shape" growth sector boundaries (GSB) which starts at the seed crystals attached at every center of the sample surface. All the samples exhibited a conical shape of trapped-field distributions when they are magnetized by 5-T FC method, which means that the samples are prepared to be magnetically single domain structures. We note that the position of the seed crystal for the 40\% Y211 sample, shown in Fig. 3(c), slightly deviated from the center, which might give the invading-fluxes anomalous behaviors, as discussed later in the paper.

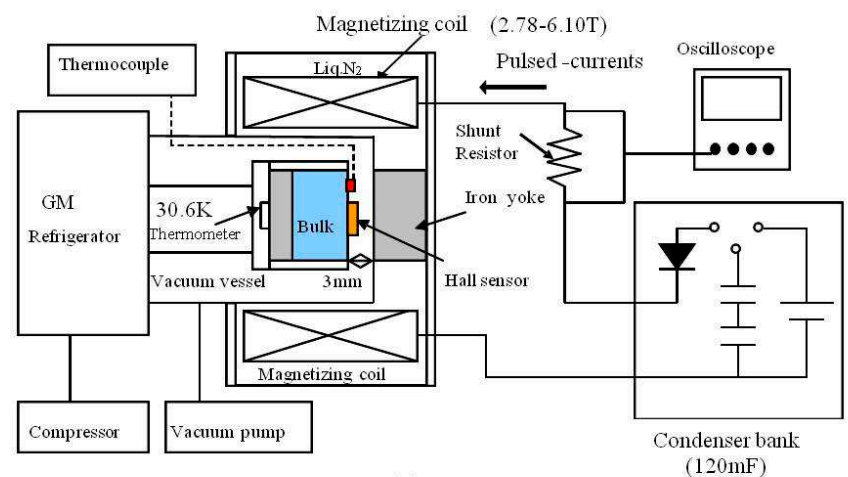

(a)

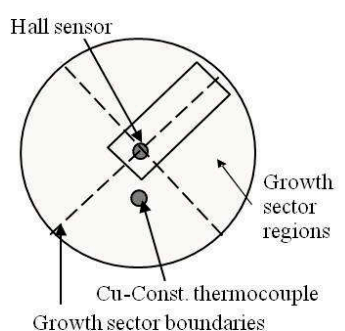

(b)

Fig. 2. Schematic illustration of the bulk magnet system equipped with GM refrigerator and the magnetizing circuit system (a), and the positions of sensors on the sample surface (b). 


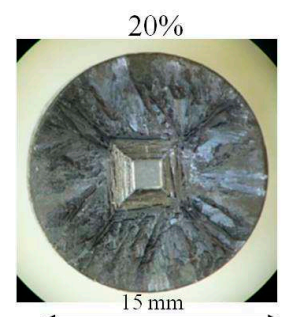

(a)

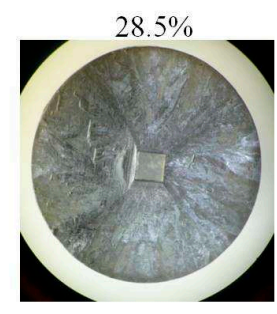

(b)

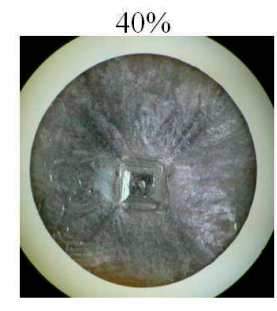

(c)

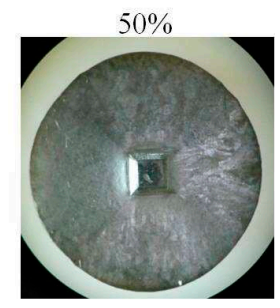

(d)

Fig. 3. Top-views of the melt-processed bulk magnet samples with various nominal Y211 contents.

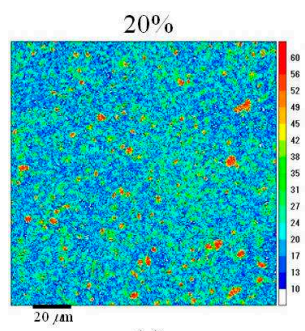

(a)

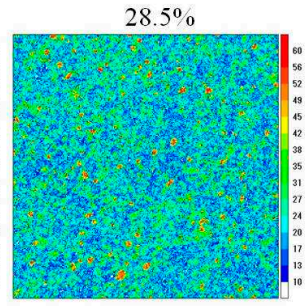

(b)

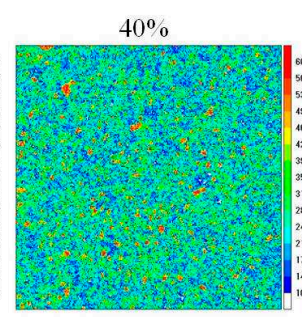

(c)

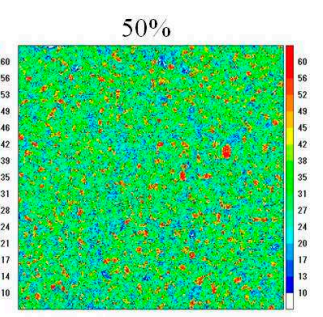

(d)

Fig. 4. Compositional mappings on Y by EPMA.

Figure 4 shows the compositional maps on $\mathrm{Y}$ which were focused on the points in the grain sector regions (GSR) at the point $4 \mathrm{~mm}$ distant from the surface center. The red spots indicate the high Y-concentration points which correspond to the Y211 particles. The spots distribute uniformly with the size of several micron-meters. The whole color of each picture certainly changed from blue to green, which means that the number of the Y211 particles obviously increases with increasing Y211 addition.

\subsection{Trapped-fields of bulk magnets after PFM}

The trapped magnetic flux densities which were measured at the center of each successive pulsed-field application are shown in Fig. 5. One sees that the starting points of the field invasions varied with each sample bearing various Y211 contents. This implies that the variation of $J_{\mathrm{c}}$ results in the different flux-invading motions into the samples. Furthermore, the number of the pinning centers which originates from the Y211 particles is apparently seen to be promoted with increasing Y211 concentration, as shown in the EPMA

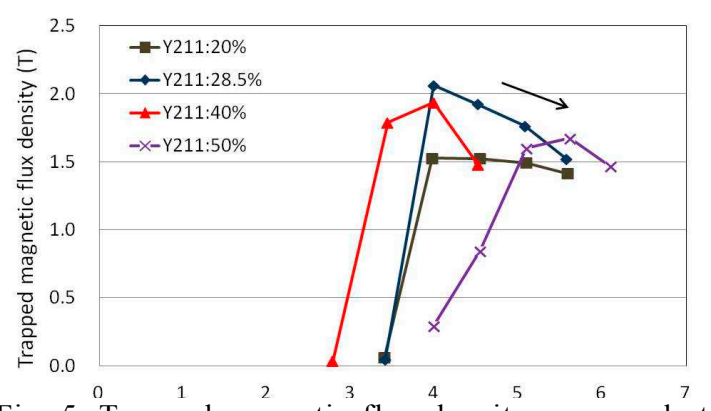

Fig. 5. Trapped magnetic flux density measured at the center of the sample surface as a function of the successive pulsed-fields. mappings. On the other, we must emphasize that every profiles shows a peak of trapped-field and following gradual decrease, as shown by an arrow in Fig. 5. This is attributed to the heat generation which occurs during the flux invasion by their motion [6]. The highest trapped field of $2.1 \mathrm{~T}$ was obtained for the sample containing $28.5 \%$ Y211. Since the distribution of Y211 particles in the sample is known to be changed by the grain growth conditions, the trapped field performances are well dependent on their fabrication conditions.

Figure 6 shows the time evolutional profiles of invading magnetic fluxes which were measured at 4.0 and $4.5 \mathrm{~T}$ pulsed-field applications as a function of Y211 contents. The magnetic fluxes invade in the sample with certain delays of several $\mathrm{ms}$ from the profiles of the applied fields both in the cases of 4.0- and 4.5-T applications. After showing peaks at around $30 \mathrm{~ms}$, these profiles gradually decreased in the same manner and resulted in the different trapped-field performances according to the amounts of fluxes which reached the center due to the differences of the flux pinning properties among the samples and corresponding heat generations. Although the magnetic fluxes behaviors are thus estimated as similar in these cases, however, the profile of $40 \%$ sample exhibited the peculiar flux motions in comparison to others. The flux started to invade at the earliest times and reached the highest peaks than those of others, showing anomalous decays at about 40 - $50 \mathrm{~ms}$ due to the severer heat generations, resulted in the inferior trapped-fields to others. The magnetic fluxes must go in and escape from the sample easier than other samples.

We defined the parameters of flux-penetration ratio $R_{\mathrm{p}}$ ( $\left.=B_{\mathrm{p}} / B_{\max }\right)$ and flux-trapping ratio $R_{\mathrm{t}}\left(=B_{\mathrm{t}} / B_{\mathrm{p}}\right)$ so as to describe the flux behaviors during the activation processes. The value of $B_{\max }$ means the highest value in the profile of the applied field. $B_{\mathrm{p}}$ expresses the highest magnetic flux density which reached the center during the profiles, corresponding to the peak of each profile. $B_{\mathrm{t}}$ means the resultant trapped-field. These parameters are 


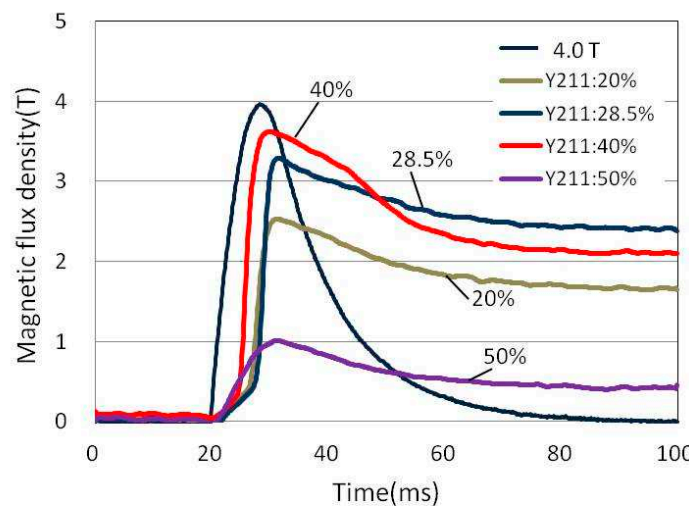

(a)

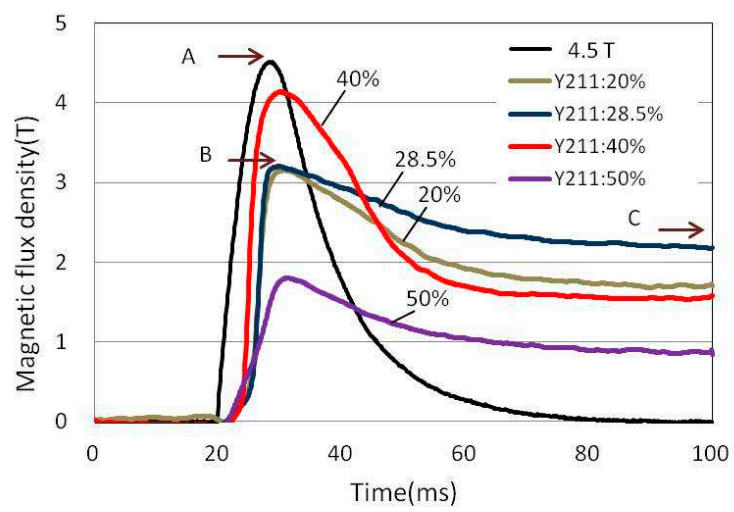

(b)

Fig. 6. Evolutional profiles of magnetic flux invasion behaviors at 4.0 (a) and $4.5 \mathrm{~T}$ (b) pulsed-field applications.

indicated by the arrows A-C in Fig. 6(b). Figure 7 shows the applied field dependence of the flux penetration as a function of Y211 content. The flux-penetration ratio $R_{\mathrm{p}}$ is enhanced with increasing applied field, showing a clear tendency of positive correlation. This implies that the high field application is necessary to obtain the high field trapping. On the contrary, as shown in Fig. 8, the flux-trapping ratio $R_{\mathrm{t}}$ drastically decreased with increasing field after showing peaks, according to the heat generation and following decay of $J_{\mathrm{c}}$. Both the $R_{\mathrm{p}}$ and $R_{\mathrm{t}}$ profiles of $40 \%$ Y211 sample exhibited the earliest flux penetration and relatively high $R_{\mathrm{p}}$ and the highest $R_{\mathrm{t}}$ at $3.4 \mathrm{~T}$. These anomalous behaviors may suggest a possible way to perform the effective high-field trappings in the low pulsed-field applications.

\subsection{Temperature measurement}

Figure 9 shows the temperature changes of each successive pulsed-field application as a function Y211 contents. Since all the data clearly coincide with a united line, we see whole the temperature rises of each sample are dominated only by the magnitudes of applied magnetic fields, and whole amount of the heat generation occurs in the thermally-insulated circumstance in the vacuum vessel. Remnant magnetic fluxes before every pulsed-field application never take any roles to suppress whole the heat generation or whole the temperature changes. This implies that the heat

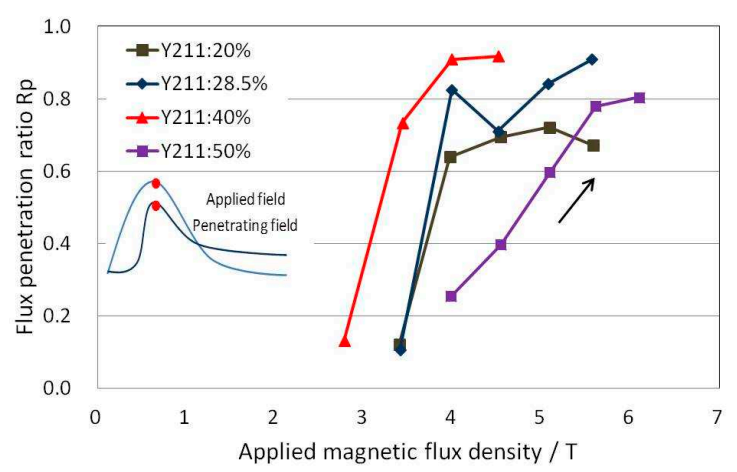

Fig. 7. Applied field dependence of the flux-penetration ratios. generation happens only at the local points on the ascending stage of the applied field, and the flux motions are locally affected by the local heating in the descending stage for the short time during the pulsed-field application. The evaluation is consistent with the anomalous rapid flux invasion and the fast decrease on the field profile of 40\% Y211 in Fig. 6.

\subsection{Discussion}

Table 1 shows the characteristics of bulk magnet samples with various Y211 contents. The 40\% Y 211 sample exhibits superior values both of the flux-penetration and flux-trapping properties. We noted that the position of the seed crystal was displaced $4 \mathrm{~mm}$ from the center. On the other as mentioned in the figures above, the flux penetration behavior of $40 \%$ Y 211 sample is different from those of other samples. Figure 10 shows the discussion on the paths of flux invasion. Since we already know that the flux invasion prefer starting at the GSR regions bearing low $J_{\mathrm{c}}$ phases to the GSB regions which were characterized as high $J_{\mathrm{c}}$ [14]. The displaced seed crystal must slightly change the arrangement of GSB and GSR, and might have led the flux preferentially through the specific paths, as shown by various arrows in Fig. 10(b). Thus, as for the sample, it is inferred that the facts of rapid flux invasions and outstanding decreases in the field profiles, as shown in Fig. 6, are attributed to the non-uniform flux behaviors

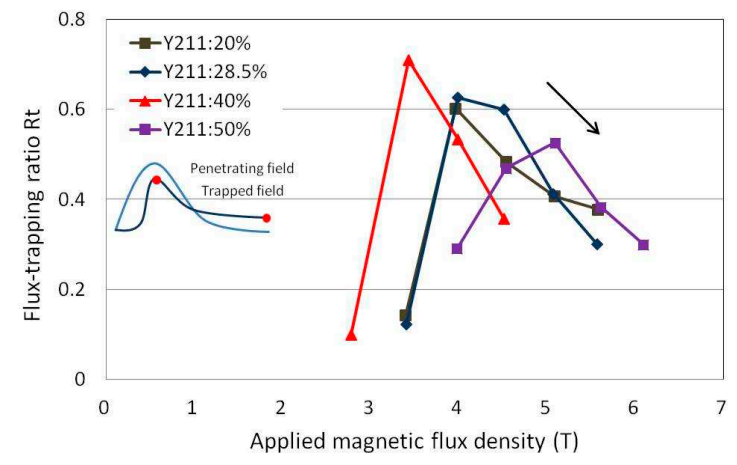

Fig. 8. Applied field dependence of the flux-trapping ratios. 


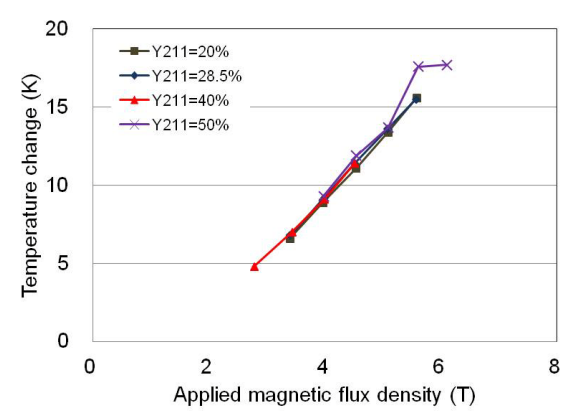

Fig. 9. Temperature change as a function of applied magnetic flux density.

and resultant local heat generations. At present, although we do not point which path the fluxes entered in the $40 \%$ Y211 sample, in order to develop the high trapped-field magnets over several $\mathrm{T}$, we believe it necessary to construct the flux paths properly in the samples and to clarify the flux behaviors in the PFM processes.

\section{CONCLUSION}

The characteristic behaviors of the magnetic flux-trapping to the melt-processed $\mathrm{Y}-\mathrm{Ba}-\mathrm{Cu}-\mathrm{O}$-based bulk magnets were precisely evaluated during the successively applied pulsed-field magnetization processes. The applied-fields at which the magnetic flux began to invade the sample increased with increasing Y211 contents, while the temperatures of the samples equally elevated with increasing applied fields. These phenomena mean that the flux pinning effect which correlated to the Y211 contents regulates the flux invasion, and the heat generation happened under the thermally insulated conditions.

The estimations on the flux-penetration ratios and flux-trapping ratios revealed that the high field application is necessary to lead the high field-trapping, while the flux-trapping drastically decreased with increasing field, according to the heat generation and following decay of $J_{\mathrm{c}}$. The flux invasion in the sample which was characterized by the displaced seed crystal position from the center of the sample surface showed us the enhanced flux-penetration and flux-trapping ratios. The facts of the rapid flux invasions and decreases at lower applied-fields than those of other samples are attributed to the preferential flux motions through the specific paths and resultant local heat generations. This suggests a possible high-field activation method with preferential flux invasion due to the different flux-pinning properties in the sample.

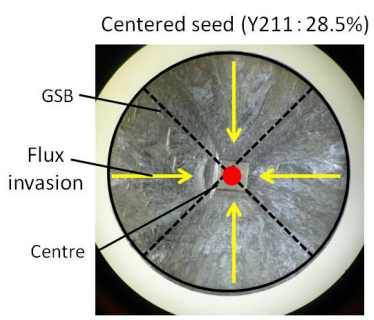

(a)

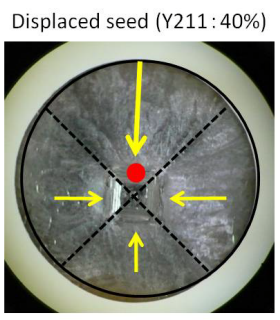

(b)
Fig. 10. Discussion on the positions of centered (a) and displaced (b) seed crystals. The arrows show the possible paths of flux invasions.

\section{References}

[1] S. Wipf, H. Laquer, IEEE Trans. on Magnetics 25, 1877-1880 (1989).

[2] G. Krabbes, G. Fuchs, P. Schatzle, S. Gruss, J. Park, F. Hardinghaus, Y. Yamashita, K. Tanabe, $A d v$. Supercond XII, Springer-Verlag, 437-440 (2000).

[3] M. Tomita, M. Murakami, Nature, 421, 517-520 (2003).

[4] T. Oka, Y. Itoh, Y. Yanagi, M. Yoshikawa, H. Ikuta, U. Mizutani, Physica C, 335, 101-106 (2000).

[5] H. Ikuta, H. Ishihara, Y. Yanagi, Y. Itoh, U. Mizutani, Supercond. Sci. Technol. 15, 606-612 (2002).

[6] Y. Itoh, U. Mizutani, Jpn J Appl Phys, 35, 2114-2125 (1996).

[7] U. Mizutani, A. Mase, K. Tazoe, H. Ikuta, T. Oka, Y. Itoh, Y. Yanagi, M. Yoshikawa, Physica C, 335, 92-96 (2000).

[8] M. Sander, U. Sutter, R. Koch, M. Klaeser, Supercond. Sci. Technol. 13, 841-845 (2000).

[9] U. Mizutani, T. Oka, Y. Itoh, Y. Yanagi, M. Yoshikawa, H. Ikuta, Applied Superconductivity, 6, 235-246 (1998).

[10] H. Fujishiro, T. Tateiwa, A. Fujiwara, T. Oka, H. Hayashi, Physica C, 445-448, 334-337 (2006).

[11] H. Fujishiro, T. Hiyama, T. Tateiwa, Y. Yanagi, T. Oka, Physica C, 463-465, 394-397 (2007).

[12] T. Oka, H. Seki, D. Ishiduka, J. Ogawa, S. Fukui, T. Sato, K. Yokoyama, A. Murakami, J. Phys. Conf. Ser., 400, 022089 (2012).

[13] T. Oka, Y. Itoh, Y. Yanagi, H. Tanaka, S. Takashima, Y. Yamada, U. Mizutani, Physica C, 200, 55-64 (1992).

[14] Y. Yanagi, Y. Itoh, M. Yoshikawa, T.Oka, H. Ikuta, U. Mizutani, Supercond. Sci. Technol. 18, 839-849 (2005).

Table I. Magnetic field-trapping characteristics of bulk magnet samples with various Y211 contents in PFM processes.

\begin{tabular}{ccccc}
\hline Y211 content & $20 \%$ & $28.5 \%$ & $40 \%$ & $50 \%$ \\
\hline $\begin{array}{c}\text { Maximum trapped } \\
\text { magnetic flux density } B_{\mathrm{T}}(\mathrm{T})\end{array}$ & 1.53 & 2.06 & 1.94 & 1.67 \\
$\begin{array}{c}\text { Flux-penetration ratio } R_{\mathrm{p}} \\
\text { Flux-trapping ratio } R_{\mathrm{t}}\end{array}$ & 0.72 & 0.91 & 0.91 & 0.80 \\
\hline $\mathrm{R}_{\mathrm{p}}=$ Maximum penetration field $B_{\mathrm{p}} /$ Maximum applied field $B_{\mathrm{max}}$ & & 0.53 \\
$\mathrm{R}_{\mathrm{t}}=$ Trapped-field $B_{\mathrm{t}} /$ Maximum penetration field $B_{\mathrm{p}}$ & & \\
\hline
\end{tabular}

